\title{
CD38 as a Therapeutic Target
}

\author{
George T Stevenson ${ }^{1}$ \\ ${ }^{1}$ Tenovus Laboratory, Southampton University Hospitals, Southampton SO16 7AD, U.K.
}

\begin{abstract}
The CD38 molecule is well represented on cell surfaces in many cases of a variety of lymphoid tumors, notably multiple myeIoma, AIDS-associated lymphomas, and post-transplant lymphoproliferations. As such, this molecule is a promising target for antibody therapy. After early disappointments, improved anti-CD38 antibodies of strong cytolytic potential have been described by 3 groups. First, a human IgG monoclonal anti-CD38 antibody raised in mice transgenic for human lg has been found to induce potent complement and cellular cytotoxicities against both myeloma cell lines and fresh harvests from myeloma marrow and leukemic blood. This antibody also exhibits the singular property of inhibiting the CD38 cyclase activity. Second, a series of CD38specific human antibodies, with high affinities and high ADCC activities against cell lines and primary cultures of myeloma, has been selected from a unique phage-display library. Finally, to enhance specificity for myeloma cells, bispecific domain antibodies targeting both CD38 and CD138 have been developed. As they lack any Fc module, these constructs rely on cytotoxicity for delivering a toxin to tumor cells. The list of candidate CD38-bearing neoplasms as targets for these antibody constructs can now be expanded to include acute promyelocytic leukemia, and possibly other myeloid leukemias, in which surface CD38 can be induced by retinoid treatment. One caveat here is that evidence has been produced to suggest that CD38 promotes pulmonary manifestations of the hazardous retinoic acid syndrome.
\end{abstract}

Online address: http://www.molmed.org

doi: 10.2119/2006-00082.Stevenson

\section{INTRODUCTION}

CD38 is a small multifunctional glycoprotein (1) widely represented on lymphoid and myeloid lineages but absent from most mature resting lymphocytes. In many lymphoid tumors-including most cases of myeloma (2), many cases of AIDS-associated lymphoma (3), and many cases of post-transplant lymphoproliferations (4) - it is present on the cell surface in amounts which render it an attractive target for therapeutic antibody.

In an early assessment, a mouse Fabhuman Fc chimeric construct, of antiCD38 specificity, efficiently mediated antibody-dependent cellular cytotoxicity (ADCC) against a CD38-displaying lymphoid cell line using human blood mononuclears as effector cells (5). Although CD38 is present on the surface of NK cells, probably the major effector population among the mononuclear cells in the assay (6), this did not seem to impair their effector function. In addition, the antibody did not inhibit the growth of erythroid or myeloid progenitors from normal bone marrow, and it seems likely that the earliest hemopoietic stem cells do not express CD38 nor other lineage commitment antigens (7). A further study reported the development of an anti-CD38 immunotoxin capable of killing human myeloma and lymphoma cell lines (8). However these early investigations did not lead to useful clinical applications. There is an urgent need among CD38-bearing neoplasms, and in multiple myeloma particularly, for new reagents to supplement present therapy. Several groups are responding with programs for developing more effective antiCD38 antibodies.
Address correspondence and reprint requests to George T Stevenson, Tenovus Laboratory, Southampton University Hospitals, Southampton SO 16 7AD, U.K.; Phone: + 44238079 6639; Fax: + 44238070 4067; e-mail: gts1@soton.ac.uk

Submitted September 29, 2006; accepted for publication December 19, 2006.

\section{HUMAX-CD38 ANTIBODY}

Paul Parren and Michael de Weers (Genmab, Utrecht, Netherlands) described a human anti-CD38 IgG1, codenamed HuMax-CD38, raised after immunizing transgenic mice possessing human, but not mouse, Ig genes. Immunofluorescent studies revealed binding of the antibody to CD8transfected Chinese hamster ovary (CHO) cells, a panel of CD38-expressing human cell lines, and freshly isolated myeloma cells. Using human blood mononuclears as effectors, HuMax-CD38 revealed potent ADCC against CD38expressing B-lymphoid and myeloma cell lines, against myeloma cells freshly isolated from patients' marrows, and against leukemic cells from a patient with $\mathrm{CD} 38^{+} / \mathrm{CD} 138^{+}$, chemotherapyresistant plasma cell leukemia. The antibody mediated complement cytotoxicity against primary myeloma-cell cultures isolated from a panel of 13 patients.

In a xenograft model in SCID mice, the antibody inhibited the outgrowth of human B-lymphoma cells, in both preventive and therapeutic settings. In a sec- 
ond xenograft model, it selectively depleted plasma cells from a human rheumatoid synovium engrafted in SCID mice. Finally, it was of considerable interest that HuMax-CD38 revealed a unique functional activity in inhibiting the CD38 ADP-ribosyl cyclase activity in target cells.

\section{ANTI-CD38 ANTIBODY}

Using a different approach to the production of fully human antibodies, Robert Friesen (MorphoSys AG, Martinsried, Germany) reported the selection of CD38-specific IgG antibodies by cellpanning strategies from a unique phagedisplay library. All the antibodies gave values for $\mathrm{K}_{\mathrm{D}}$ in the low nanomolar range and recognized myeloma samples by flow cytometry and immunohistochemistry. In ADCC assessments CD38expressing cell lines and primary myeloma cultures from patients were killed efficiently, whereas in clonogenic assays marrow progenitor cells appeared not to be affected. Efficacy in vivo was shown by reduced tumor growth in a SCIDmouse xenograft model.

\section{BISPECIFICITY YIELDING INCREASED SELECTIVITY}

One practical problem, which might be encountered in applying anti-CD38 therapy, is the breadth of occurrence of the molecule on lymphoid, myeloid, and epithelial cells, especially following cell activation. Steve Holmes (Domantis Ltd, Cambridge, U.K.) described an antibody derivative aimed at increasing specificity by targeting two surface molecules on myeloma cells, CD38 and CD138. This combination is thought to be exquisitely specific for myeloma cells. Moderate affinity monomeric domain antibodies (dAbs) specific for each antigen have been isolated using phage display. Combining the dAbs as a dualtargeting molecule yields constructs with high avidity for myeloma cells expressing both antigens, but binding only weakly to cells expressing only one antigen. The combined $\mathrm{dAbs}$ are conjugated to a cytotoxic agent, with the resulting construct delivering a cytotoxic payload on being internalized.

\section{INDUCING EXPRESSION OF CD38}

Kapil Mehta and Yin Gao (University of Texas M.D. Anderson Cancer Center, Houston, U.S.A.) raised the possibility of expanding the list of CD38-bearing neoplasms to include acute promyelocytic leukemia, and possibly other myeloid leukemias. All-trans retinoic acid (RA), as well as being a leading form of therapy for acute promyelocytic leukemia (APL), is a potent and selective inducer of cell-surface CD38 in myeloid leukemia cells at low concentrations. This CD38 can function as a therapeutic target, as revealed by a demonstration that the combination of RA and an anti-CD38gelonin immunotoxin induced a synergistic killing of leukemia cells_-both leukemia cell clones and blasts from patients with myeloid leukemia (9).

The optimism resulting from this cellular manipulation must however be tempered by evidence that CD38 might be involved in the RA syndrome, a complex form of cardiorespiratory distress which occurs in about $25 \%$ of APL patients treated with RA (10). The maturation of APL cells induced by RA induced the expression of interferon- $\gamma$ and interleukin$1 \beta$, plus the expression of CD38. The latter was judged to promote the strong attachment of leukemic cells to endothelial cells, judged by the fact that the binding was blocked either anti-CD38 or soluble recombinant CD38. A likely ligand on the endothelial cells is CD31. Both cellular adhesion and cytokine production are probably important in the pathogenesis of the RA syndrome. There is clearly great scope for further molecular studies in this area of oncology and induced differentiation.

\section{REFERENCES}

1. Deaglio S, Mehta K, Malavasi F. (2001) Human CD38: a (r)evolutionary story of enzymes and receptors. Leuk. Res. 25:1-12.

2. Chan CS, Wormsley SB, Pierce LE, Peter JB, Schechter GP. (1990) B-cell surface phenotypes of proliferating myeloma cells: target antigens for immunotherapy. Am. J. Hematol. 33:101-9.
3. Hoffmann C et al. (2005) AIDS-related B-cell lymphoma (ARL): correlation of prognosis with differentiation profiles assessed by immunophenotyping. Blood. 106:1762-9.

4. Rochford R, Hobbs MV, Garnier JL, Cooper NR, Cannon MJ. (1993) Plasmacytoid differentiation of Epstein-Barr virus-transformed B cells in vivo is associated with reduced expression of viral latent genes. Proc. Natl. Acad. Sci. U. S. A. 90:352-6.

5. Stevenson FK et al. (1991) Preliminary studies for an immunotherapeutic approach to the treatment of human myeloma using chimeric anti-CD38 antibody. Blood. 77:1071-9.

6. Ojo E, Wigzell H. (1978) Natural killer cells may be the only cells in normal mouse lymphoid cell populations endowed with cytolytic ability for antibody-coated tumor target cells. Scand. J. Immunol. 7:297-306.

7. Verfaillie CM, Miller JS. (1994) CD34+/CD33cells reselected from macrophage inflammatory protein 1 alpha+interleukin-3-supplemented "stroma-noncontact" cultures are highly enriched for long-term bone marrow culture initiating cells. Blood. 84:1442-9.

8. Goldmacher VS et al. (1994) Anti-CD38-blocked ricin: an immunotoxin for the treatment of multiple myeloma. Blood. 84:3017-25.

9. Mehta K, Ocanas L, Malavasi F, Marks JW, Rosenblum MG. (2004) Retinoic acid-induced CD38 antigen as a target for immunotoxinmediated killing of leukemia cells. Mol. Cancer Ther. 3:345-52.

10. Gao Y, Camacho LH, Mehta K. (2006) Retinoic acid-induced CD38 antigen promotes leukemia cells attachment and interferon- $\gamma /$ interleukin- $1 \beta$ dependent apoptosis of endothelial cells: Implications in the etiology of retinoic acid syndrome. Leuk. Res. in press. 